\title{
Percepciones Ambientales de los habitantes del Parque Natural Páramo de Miraflores en Colombia. Estudio de caso en la vereda Las Mercedes, municipio de Garzón
}

\author{
Environment Understandings of the Inhabitants of the Miraflores \\ Paramonatural Park in Colombia. Case study in the Mercedes \\ village in Garzon city
}

Pavel Tovar Lizcano

Magister en Ecología y Gestión de Ecosistemas Estratégicos. SED Huila. Universidad Surcolombiana. Neiva. Colombia. paveltl@gmail.com

Alfredo Olaya Amaya Phd. Ingeniería Área Recursos Hídricos. Universidad Surcolombiana alfredo.olaya@usco.edu.co

\section{Resumen}

En Colombia los ecosistemas estratégicos incluyen zonas rurales, algunas densamente pobladas, su conservación tiene el reto de una gestión sistémica que incluya la dinámica social, económica y cultural de sus pobladores, y que además permita el desarrollo rural sostenible. Diversos autores señalan la necesidad de tener una visión biocultural en la conservación de los Parques Naturales, sin embargo, sus planes de manejo se limitan a regular o restringir las acciones de los pobladores sin tener en cuenta las percepciones que ellos tienen del área objeto de conservación.

En consecuencia, el presente trabajo estudió la manera como las percepciones ambientales de los pobladores del Parque Natural Páramo de Miraflores influyen en su conservación, para esto se aplicaron métodos de la investigación acción participación en combinación con técnicas de identificación y jerarquización de problemas. Se encontraron diferencias contrastantes entre las percepciones de los pobladores del Parque, la visión institucional y la postura de los académicos.

Además, se expone que los siguiente tres problemas: La incipiente articulación entre las instituciones que forman parte del Sistema Regional de Áreas Protegida, el Sector Educativo, las ONG's ambientales y los habitantes de la vereda Las Mercedes; El desconocimiento de la importancia eco sistémica, económica, y social de la biodiversidad presente en el Parque; Y el no aprovechamiento de las oportunidades de desarrollo sostenible, son prioritarios para su atención y demuestran la importancia de los aspectos sociales y culturales en la gestión orientada a la conservación eco sistémica de territorios que se encuentran poblados.

Palabras claves: Gestión de ecosistemas estratégicos, Percepciones ambientales, Parque Natural Páramo de Miraflores, Pobladores locales.
Abstract

In Colombia the strategic ecosystems include rural zones, some of the highly populated. It's conservation has the challenge of being a systemic management that includes the social, economy and cultural dynamics of its inhabitants and also allows the sustainable cultural development. Diverse authors outline the need of having a bio-cultural sight in the Natural Parks conservation. However, it's management plans limit to regulate or restrict the inhabitants actions without taking into account the perceptions that they have from the area that is being conserved.

As a result, this research studied the way as environment perceptions of the Miraflores Paramo Natural Park inhabitants influence in its conservation, for this, investigation methods of the actionparticipation were applied in combination with identification techniques and issue prioritization. Some contrast-differences were found between the Park inhabitants perceptions, the institutional vision and the academic community posture.

Keywords: Strategic ecosystems management, Environment perceptions, Miraflores Paramo Natural Park, Local Inhabitants.

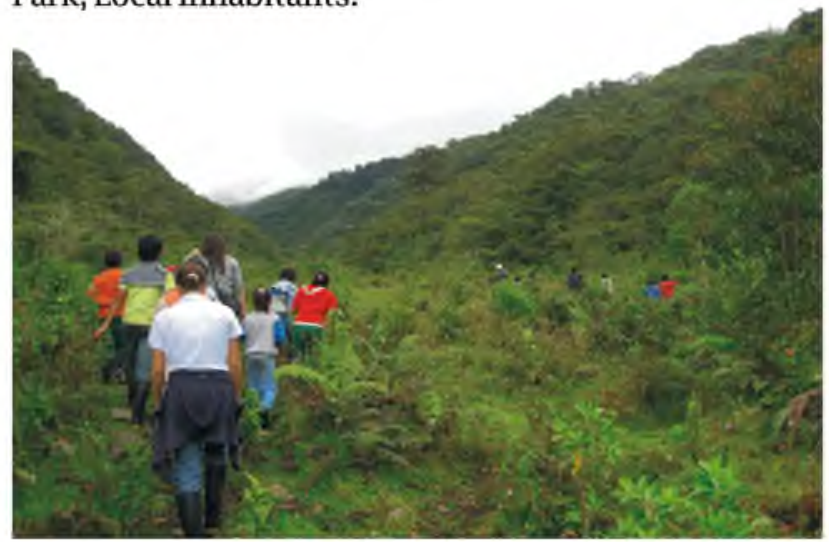




\section{Zusammenfassung}

In Kolumbien strategischen Ökosysteme gehören ländliche Gebiete, einige sogar dicht besiedelt. Ihre Erhaltung $\mathrm{zu}$ einem systemischen Ansatz ist nicht einfach. Ausserdem der sozialen, wirtschaftlichen und kulturellen Dynamik der Bewohner werden angefochten, und auch eine nachhaltige ländliche Entwicklung ist nicht $\mathrm{zu}$ ermöglichen. Mehrere Autoren weisen auf die Notwendigkeit einer Vision biokulturellen Erhaltung der Naturparks, aber Bewirtschaftungspläne sind lediglich nicht zu regulieren oder es sind keine Aktionen der Menschen zu beschränken. Unabhängig von den Wahrnehmungen sind der Fläche unter Naturschutz nicht stark.

\section{Introducción}

Las Áreas Protegidas son espacios dedicados a la conservación de los recursos naturales que se han identificado como valiosos en términos de biodiversidad y oferta de bienes y servicios ambientales. El Parque Natural Cerro Páramo de Miraflores (PNCPM) que incluye algunos territorios densamente poblados y al estar vinculado con la Amazonía Colombiana, la Zona Andina y la Cuenca Interandina del Magdalena, se hace poseedor de ecosistemas de gran riqueza en términos de biodiversidad (CAM, 2006, 2005), (Alcaldía Municipal de Garzón, 2007), (Ingenieros y Biólogos Ltda, 2006), (Recuperam Ltda, 2003), (Consejo Municipal de Gigante, 2000).

Este Parque Natural es un ecosistema estratégico para la subregión centro del departamento del Huila, puesto que produce el agua que abastece los acueductos de los municipios de Algeciras, Garzón y Gigante, importantes factorías piscícolas y distritos de riego para la producción agrícola (Ingenieros y Biólogos Ltda, 2006), (Recuperam Ltda, 2003). Por lo anterior, el Parque en concordancia con los conceptos de Márquez (2003) se valora como una área donde se concentran funciones naturales, de las cuales dependen de manera significativa bienes y servicios ecológicos vitales para el mantenimiento de la sociedad.

Además, Olaya, Vargas y Rubiano (2005), mediante el análisis de 25 criterios ecológicos y socioculturales, reconocieron la importancia estratégica de Miraflores como Parque Natural y determinaron su relevancia en términos de diversidad natural, interacción con ecosistemas andinos y amazónicos, producción de agua para consumo humano, y agricultura y producción pecuaria. De igual manera, el Ministerio de Ambiente, Vivienda y Desarrollo Territorial, considera ecosistemas estratégicos los páramos, sub-páramos, los nacimientos de agua y las zonas de recarga de acuíferos (Decreto 2372, 2010), características que cumple bien el PNCPM.

En Colombia, la principal estrategia para la conservación de la biodiversidad se basa en el establecimiento de áreas protegidas aisladas y con intervenciones humanas controladas o prohibidas. Este enfoque desconoce que los procesos biológicos tienen diferentes escalas espaciales y temporales, así como también, que la mayoría de estas áreas se encuentran pobladas. En consecuencia, diversos autores señalan que la gestión de los recursos naturales en Colombia se encuentra en una situación crítica (Morales, 2007), (Zambrano, 2007), (Arango, 2005), (Kattan, 2005), (Martínez, 2004). Un análisis del plan de manejo del PNPM muestra la misma tendencia al concebirlo como un sistema que no interactúa con otras áreas ni con la cultura de sus pobladores, que necesitan recursos y realizan transformaciones (Ingenieros y Biólogos Ltda, 2006), (CAM, 2005).

La situación anteriormente descrita concuerda con la visión histórica que considera a los pobladores de las áreas protegidas como una amenaza hacia aquellos valores que incentivan la declaración de dichas áreas. Esta circunstancia ha generado numerosos debates en torno a la manera de administrar y las posibilidades de desarrollo de estas áreas, planteando la necesidad de un viraje hacia formas de gestión interactivas que reconozcan las necesidades de desarrollo de la población residente en su interior como una de sus prioridades (Escobar, 1999).

Otros autores plantean que los sistemas sociales forman parte de la biodiversidad, por ende, no somos sus simples observadores o custodios, sino que coexistimos dentro de su trama; por esto, es necesario pasar de una concepción de biodiversidad desde un plano biológico y ecológico, a uno más complejo e integrador que reconozca al humano y su desarrollo cultural (Vásquez, 2007), (Nielsen, 2007), (CDB,2004), (Arango, 2005), (Martínez, 2004) y (González y Galindo, 1999).

Estas situaciones constituyen un reto para la gestión ambiental y plantean el desarrollo de nuevos modelos que incluyan los aspectos anteriormente mencionados. En efecto, existen marcos conceptuales de integración que surgen de las ciencias sociales y ecológicas, que muestran la naturaleza social de los conflictos entre los seres humanos y los recursos naturales, integrando aspectos culturales, económicos y ecológicos para así mejorar la comprensión de los conflictos relacionados con la biodiversidad (Haberl, 2009), (White, Fischer, Marschall, Travis, Webb y Falco, 2009) y (Escobar, 1999) (Zimmermann, 1995)

Este tipo de comprensión de la biodiversidad considera que las personas no son independientes de su ambiente, así como tampoco lo es el ambiente de las personas que lo viven, lo interpretan y modifican (Márquez, 2003), (González y Galindo, 1999), (Zimmermann, 1995). De este modo, los estudios socio-ambientales se constituyen en un medio importante para recolectar información sobre la 
dinámica en los parques naturales habitados (Sheil, 2004). Dentro de estos estudios, las percepciones ambientales entendidas como la forma en que cada individuo y la comunidad de la que hace parte aprecian, valoran y entienden su entorno, influyen de manera importante en la toma de decisiones del ser humano sobre el ambiente que le rodea (Fernández, 2008), (González y Galindo, 1999). Es decir, la relación entre el ser humano y el ambiente es en gran parte el reflejo de sus percepciones ambientales y es aquí donde radica la importancia de conocerlas para lograr una gestión ambiental más eficiente.

En este orden de ideas, es posible determinar que dichas percepciones se derivan de la actitud que concibe la persona como sujeto que se forma en un entorno que lo influye, con sus normas, modelos y valores (Salazar, 2000), (González y Galindo, 1999), es decir, se encuentran ligadas al afecto, el conocimiento y la conducta que se establece frente al entorno (Zimmermann, 1995), lo que muestra una relación racional entre actitudes y conducta. De acuerdo con esto y según Gallego (2000), lo actitudinal está constituido, al menos, por los componentes cognoscitivos, afectivo, intencional y comportamental.

En consecuencia, se consideró importante realizar una investigación a partir del siguiente interrogante: ¿Cuáles son las percepciones ambientales que tienen los habitantes de la vereda Las Mercedes de Garzón como pobladores del Parque Natural Cerro Páramo de Miraflores?

\section{Metodología}

\section{Área de estudio}

EI PNCPM se encuentra en ubicado en el flanco occidental de la Cordillera Oriental de la subregión centro del departamento del Huila Colombia, y forma parte de los municipios de Algeciras, Garzón y Gigante del departamento del Huila, Colombia (Figura 1), con un área aproximada de 35.644 hectáreas. La vereda Las Mercedes se encuentra entre los 2000 y 3000 metros de altura con predominancia de clima frío y muy húmedo que oscila entre $12-18^{\circ} \mathrm{C}$, y una precipitación promedia anual de 2000 - $4000 \mathrm{~mm}$ (Alcaldía Municipal de Garzón, 2007,15).

Figura 1. Ubicación Parque Natural Cerro Páramo de Mirraflores
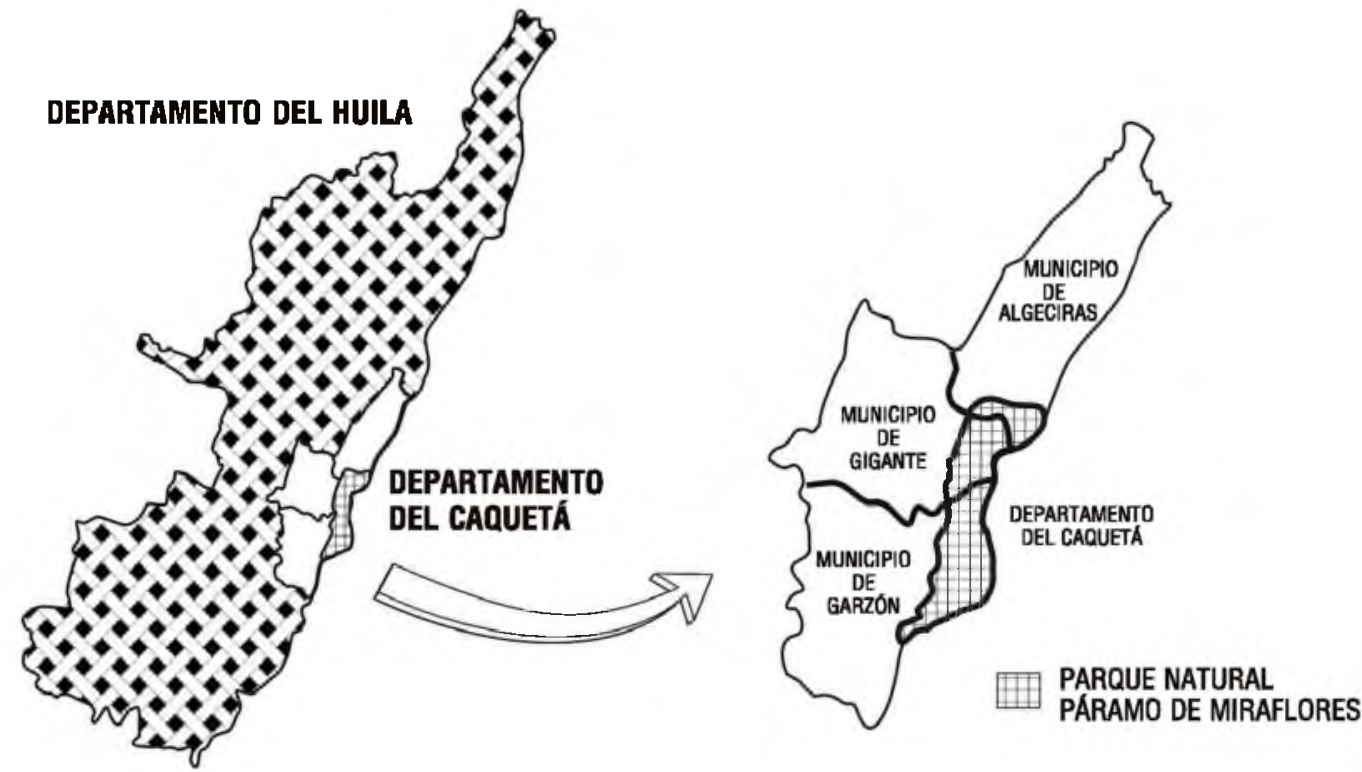

Métodos

El estudio de las percepciones ambientales de los habitantes del Parque Natural y los procesos de conservación que se realizan al nivel institucional y académico se realizó entre los años 2009 y 2011. Para lograrlo se emplearon herramientas metodológicas de la investigación acción participación, aplicadas en un estudio de caso con los pobladores de la vereda Las Mercedes. Varios criterios fueron tenidos en cuenta para la selección de esta vereda, entre estos, se tiene que su territorio se encuentra totalmente inmerso dentro del área del Parque Natural y va desde la zona de amortiguamiento hasta la zona de protección estricta (Ingenieros y Biólogos Ltda, 2006), que además es una de las veredas más pobladas del Parque Natural, con presencia en el año 2006 de 776 unidades familiares y dos grandes asentamientos humanos (Alcaldía Municipal de Garzón, 2007). Con dichos pobladores se establecieron grupos focales, se aplicaron entrevistas en profundidad y se realizó la valoración de criterios ecológicos y socioculturales propuestos por el Grupo ECOSURC de la Universidad Surcolombiana, (Castillo, 2005), (Olaya y Sánchez, 2005), (Olaya y Sogamoso, 2003). 
La metodología de grupo focal señala que cada uno de estos debe estar conformado por un número reducido no superior a 10 personas y además debe ser lo más heterogéneo posible, puesto que consiste en un mecanismo de discusión profunda y dirigida que ha demostrado su validez en el campo del desarrollo local, al facilitar la identificación de las relaciones y procesos sociales, y la construcción del escenario de cada uno de los actores (Scott, 2011), (Bertoldi, 2006). También cabe destacar que la técnica grupo focal permite la revelación de las singularidades presentes en la complejidad de los contextos culturales (Ressel, 2008), puesto que permite evaluar las preferencias de las personas frente a los recursos naturales (Powe, Garrod y Mcmahon, 2005), (Zimmermann, 1995).

Se establecieron dos grupos focales con los habitantes de la vereda Las Mercedes, uno con pobladores de la parte alta de la vereda que corresponden a las zonas de conservación estricta y de recuperación ambiental para la protección del Parque Natural y otro con pobladores de la parte baja de dicha vereda que corresponde a las zonas de amortiguación, de desarrollo socioeconómico con restricciones moderadas y mayores del Parque Natural (Ingenieros y Biólogos Ltda, 2006).

Cada grupo fue integrado por diez personas de diferentes edades, sexos, actividad económica y nivel académico. Con estos grupos se desarrollaron discusiones basadas en los principios del enfoque por ecosistemas del Convenio sobre la Diversidad Biológica (CDB, 2004), se trataron los aspectos cognoscitivos, afectivos, intencionales y comportamentales de cada uno de los participantes con respecto al PNCPM. Con los mismos grupos también se desarrolló la valoración de criterios ecológicos y socioculturales del grupo ECOSURC expuestos en el cuadro uno (Olaya y Sánchez, 2005). De este modo se logró su mayor valor metodológico, debido a que se permitieron interacciones entre múltiples perspectivas sobre la aceptación pública de las decisiones y procesos de gestión (Kallbekken y Aasen, 2010), (Xenariosa y Tziritis, 2007), (Powe, Garrod y Mcmahon, 2005), (Gallego, 2000), (Salazar, Lara, Guido, Toro y Obando, 2000).

La entrevista en profundidad se aplicó a 23 personas, entre las cuales se tienen funcionarios de entidades que tienen injerencia en el Parque como es el caso de la Corporación Autónoma Regional del Alto Magdalena (CAM), como autoridad ambiental, de la administración municipal de Garzón, como autoridad territorial, de la Institución Educativa con sedes en el área de estudio, presidentes de las juntas de acueducto y acción comunal, como organizaciones comunitarias que velan por el bienestar de los habitantes locales y presidentes de las ONG's ambientales, como entidades que realizan actividades pro-conservacionistas en dicho Parque. Esta herramienta metodológica es más flexible y abierta que los cuestionarios estructurados al consistir en un dialogo dirigido que pretende indagar y captar la experiencia subjetiva del entrevistado (Cardona, 2005).

\section{Resultados y Discusiones}

Percepciones ambientales

Se debe partir del siguiente hecho, los habitantes del PNCPM tienen estilos de vida tradicionales con una dependencia habitual de los recursos biológicos, son minifundistas de largas tradiciones familiares, que de manera paulatina fueron dividiendo y heredando las grandes fincas, con cultura agrícola que mantienen sistemas productivos de hortalizas y verduras pero de manera especial en el cultivo del café, seguido de ganadería acompañado de la cría de gallinas, pollos y cerdos. Entre los años 1990 y 2011 han direccionado sus tierras hacia el establecimiento de frutales de clima frío, como la mora, granadilla y lulo. La economía familiar es de subsistencia, muchas personas trabajan de jornaleros y tienen sus necesidades básicas insatisfechas; cocinan con leña que es extraída de los relictos de bosques que tienen en sus fincas y en ocasiones de la parte alta del Parque.

\section{Aspectos Cognoscitivos}

Los pobladores muestran escaso conocimiento sobre el parque natural, en este sentido, piensan que el PNCPM fue creado para proteger las aguas de las quebradas que se usan en los municipios de Garzón, Gigante y Algeciras, que posteriormente llegan al río Magdalena. Además reconocen que en este parque nacen muchas quebradas del departamento de Caquetá y del río Orteguaza en el mismo departamento.

En términos de biodiversidad, los pobladores del PNCPM reconocen la existencia del Oso de Anteojos (Tremarctosornatus), Dantas (Tapirussp), venados, culebras, aves como el Gallito de Roca (Rupícola peruviana), Armadillos, Guaras (Dasyproctapuncnata), Cusumbo (Nasuasp), Ardillas (Sciurussp), Águilas, Micos, Tigrillos (Leopardussp) y Zorros (Cerdocyonsp) en la parte alta, junto con el reporte de avistamientos esporádicos de un oso muy grande que denominan "oso real" (que no lo reconoce como oso de anteojos) y que desde el año 2000 no se volvió a ver.

En cuanto a la flora reportan el conocimiento de orquídeas, roble, caucho y variedad de árboles, de manera específica se refieren al comino (Anibasp) y otros árboles de maderas finas fueron acabados por los madereros y expresan que ahora prácticamente ya no existen maderas buenas en la zona. Otro factor negativo fueron las instrucciones dadas por el Comité de Cafeteros, entidad que sugirió tumbar todos los árboles que daban sombra a los cafetales en las fincas, situación que causó la erradicación de los caimos (Chrysophyllumsp), guamos (Inga $s p$ ) y palma boba (helecho arbóreo) de toda la parte baja del parque y que ahora el mismo Comité está incentivando el cultivo de café bajo sombrío.

Es posible que este poco conocimiento con respecto a la importancia del ecosistema estratégico se deba a las deficiencias en los mecanismos de comunicación e interacción establecidos entre las instituciones y las 
comunidades, es así que los pobladores manifiestan "Dicen que hay que cuidarlo pero no sabemos ipor qué?", "Es que no nos explican las cosas, sólo vienen y dicen qué hicieron y se van", "No sabemos qué función cumplimos dentro de nuestras fincas", "Cuando colocaron los mojones no socializaron nada, nos reunieron para decirnos que los habían colocado", "Nunca hemos recibido nada por cuidar el parque", "La exigencia es sólo para nosotros mientras que los del pueblo no aportan nada". Esto entre otras situaciones, ha generado que las entidades como el municipio de Garzón y la CAM pierdan credibilidad en los habitantes del parque.

Pero además es importante señalar que el sector educativo también tiene injerencia en el desconocimiento del parque natural. La Institución Educativa Agropecuario del Huila, que ejerce presencia en varias veredas que se encuentran en el PNCPM ha identificado problemas ambientales relacionados con la ampliación de la frontera agrícola, el inadecuado manejo de los pesticidas en especial en el cultivo del lulo, el incorrecto manejo de los residuos sólidos (problemática en la cual han centrado los PRAES) y de los subproductos del procesamiento del café cuando hay cosecha, que fluyen de manera directa hacia los arroyos y quebradas. Los funcionarios de dicha institución educativa catalogan a los habitantes de la región en dos grupos, uno mayoritario que no se encuentra interesado en la conservación del PNCPM y otro muy pequeño que sí está comprometido con su protección; esta situación la atribuyen al desconocimiento y falta de cultura ambiental.

En este sentido, la Institución Educativa, ha realizado trabajos de formación comunitaria en temas de conservación a través del proyecto de trabajo social y en el plan de estudios de dicha institución. Pero el programa de formación comunitaria en el año 2011 fue desmontado por indicaciones de la Secretaría de Educación Departamental del Huila ${ }^{1}$. Lo anterior demuestra la profunda desarticulación que existe entre los sectores educativo y ambiental que se da a nivel de las autoridades que los regula, que para el caso del PNCPM es la Secretaría de Educación Departamental del Huila y la CAM.

\section{Aspectos intencionales y comportamentales \\ Entre los usos que hacen los pobladores de los relictos de bosques presentes en sus fincas, es la extracción de leña para la cocción de alimentos y para obtener estantillos para cercas; también emplean la guadua para cubrir las necesidades en construcciones rurales, pero se muestran preocupados por la disminución de los guaduales.}

Frente a la fauna presente, expresan que tienen problemas ocasionales con las ardillas, loras, águilas, zorros y guaras que van a la parte baja a comerse los granos de maíz, frutales, aves de corral, plátanos y yuca. Para afrontar estas situaciones realizan diversas actividades como poner muñecos entre los cultivos, quemar azufre en los bordes o simplemente permitir que causen cierto nivel de daño en los cultivos. Aquí se observa que algunos pobladores locales tienen costumbres de caza pero al tiempo gustan de la presencia de animales en sus fincas. De manera adicional argumentan que el establecimiento de grandes cultivos de lulo ha contaminado mucho la zona, y que es común que aves e insectos aparezcan muertos cuando se realizan actividades de fumigación en dichos cultivos.

En lo relacionado con la administración del parque natural, declaran que se realizan prácticas poco adecuadas en las fincas compradas para la conservación por el municipio de Garzón; lugares en los cuales se han realizado reforestaciones con plantas no nativas cuyo cuidado no ha garantizado su prosperidad. En este sentido, explican que si se emplearan plantas de la zona, la reforestación sería más rápida y efectiva porque estas plantas crecen sin problemas.

Dentro de los inconvenientes generados por la declaratoria del área protegida argumentan que la CAM impone pero nunca presenta alternativas de solución, dicen: "La CAM viene y molesta pero nunca hemos recibido beneficios de ellos". También sus fincas han sido desvalorizadas. Así mismo, denuncian que se presentan inconvenientes con las fincas que son compradas para conservación, puesto que luego son abandonadas, facilitando la tala de los bosques remanentes en estas fincas. En el mismo sentido Recuperam (2003), el Instituto para la Sostenibilidad del Desarrollo (2007), los funcionarios de la CAM y del Municipio de Garzón entrevistados, sostienen que el déficit económico para la ejecución del plan de manejo del parque natural es un gran inconveniente.

Las situaciones anteriormente descritas muestran la incapacidad de las autoridades ambientales para lograr una adecuada administración y que en este sentido los pobladores del parque pueden contribuir de manera significativa a tener un mayor protagonismo en su conservación, lo cual sería posible en la medida que se alcance una mayor apropiación del territorio; pero este protagonismo es difícil de lograr cuando los procesos de conservación tienden a la expropiación del territorio.

Por estas razones los habitantes del parque concluyen que la declaratoria del parque natural beneficia a muchas personas, menos a ellos, puesto que sus posibilidades laborales han sido menguadas, al considerar que la disminución en la caza, la extracción de madera que demanda mano de obra y la restricción a la ganadería en zonas con buenos forrajes, son acciones que han favorecido la conservación, pero ha perjudicado sus economías familiares; sin embargo, dicen estar interesados en seguir viviendo allí.

1 - ENTREVISTA con Miguel Rodríguez, Rector Institución Educativa Agropecuario del Huila. Garzón, Febrero 17 de 2011. 


\section{Aspectos Afectivos}

En concordancia con Recuperam Ltda. (2003), se encontró que los pobladores tienen gran arraigo a la tierra, sus fincas, sus amigos y vecinos, de sus tradiciones agrícolas y pecuarias de las cuales reconocen que muchas de ellas pueden estar equivocadas, pero que de igual manera no conocen otras formas de producir. Sin embargo, algunos manifiestan que quisieran vivir en otra parte porque la declaratoria del PNCPM les ha afectado su economía familiar.

Pero también reconocen ciertos beneficios de la existencia del área protegida como son las condiciones climáticas que consideran muy buenas para sus cultivos, y para su gozo personal, la posibilidad de poder contemplar las aves y tener en sus fincas plantas que emplean de manera cotidiana como son hortalizas, verduras y plantas medicinales. Sin embargo, esperan obtener beneficios directos del parque en el futuro.

Los habitantes de la vereda Las Mercedes argumentan que se han sacrificado para conservar el parque natural, y que en este sentido se está dando un cambio en ellos, pero también reclaman la necesidad de proyectos productivos que los comprometa con su cuidado como es el caso del ecoturismo y agroturismo, con los cuales podrían aprovechar la riqueza paisajística de la zona. También exponen la necesidad de dos proyectos forestales, uno para el establecimiento de bosques para leña y así suplir sus requerimientos energéticos en la cocción de alimento, y el segundo, un cultivo de guadua para cubrir la escasez de materiales para construcciones rurales. Y por último, necesitan proyectos que les permita mejorar la habitabilidad de sus viviendas como la instalación de baterías sanitarias y de estufas eco eficiente; en este sentido la CAM ha desarrollado un proyecto de estufas eco eficiente.

De igual manera, expresan que necesitan preparación técnica para el manejo adecuado del entorno y establecimiento de cultivos limpios, acompañada de un subsidio de producción limpia. También comentan tener necesidades de capacitaciones en aprovechamiento sostenible y transformación de los recursos naturales como cultivos de flores, desarrollo de artesanías con productos forestales como semillas de árboles, en trabajo comunitario, apoyo en la comercialización de sus productos y capacitación en conocimiento del parque; tal como lo expresa Nidia Calderón, quien en el año 2011 era la presidenta de la Junta de Acción Comunal de la vereda Las Mercedes, "Cuando uno no conoce, no valora y no cuida". Por estos aspectos se deben plantear alternativas económicas sostenibles a los pobladores del área protegida como una posibilidad de compartir los beneficios que se derivan de la biodiversidad allí presente.

Aunque los pobladores reconocen la importancia del PNCPM, le es indiferente la categoría del mismo, es decir, si es área protegida es de carácter nacional o regional; frente a esto, no les interesa cual entidad es la que ejerce autoridad, siempre y cuando tenga en cuenta la parte social, y consideran que más que las instituciones son ciertos funcionarios que por su cercanía a ellos los han apoyado en ciertas iniciativas y que a pesar de ser muy pocas las reciben con beneplácito.

\section{Problemáticas identificadas}

Mediante las opiniones o juicios de concurrencia y de contradicción que se presentaron entre los pobladores del parque y los funcionarios entrevistados, se logró establecer las principales problemáticas y oportunidades que se deberían abordar para mejorar el plan de manejo ambiental.

En el reconocimiento y valoración que hacen los pobladores del PNCPM según los criterios desarrollados por ECOSURC (Cuadro 1) (Olaya y Sánchez, 2005), se determinó que de los 25 criterios propuestos, los de mayor jerarquía son: 1) Agua para consumo humano (C4); 2) Turismo y recreación (C14); 3) Depuración natural de aguas (C9); 4) Agua y suelos para la agricultura y la ganadería (C8); 5) Agua para uso industrial (C12). Es importante resaltar que cuatro están relacionados con el servicio hidrológico del ecosistema y que el segundo lugar de importancia para los pobladores es el potencial turístico.

Cuadro 1. Adaptado de OLAYA, Alfredo y SÁNCHEZ, Mario. Significado ecológico y sociocultural de los ecosistemas estratégicos del Huila. En: OLAYA, Alfredo y SÁNCHEZ, Mario, editores. Del Macizo Colombiano al Desierto La Tatacoa: la ruta del río Magdalena en el Huila. Neiva: Universidad Surcolombiana, 2005. pp. 17-29.

\begin{tabular}{|l|l|}
\hline \multicolumn{2}{|l|}{ CRITERIOS } \\
\hline Codigo(Ci) & Nombre \\
\hline C1 & Asentamientos humanos \\
\hline C2 & Paisaje v creación artistica y literaria \\
\hline C3 & Pesca y acuicultura \\
\hline C4 & Agua para consumo humano \\
\hline C5 & Patrimonio histórico y araueológico. \\
\hline C6 & Control geopolítico y milltar. \\
\hline C7 & Emblemas municipal. \\
\hline C8 & Agua y suelos para la agricultura yla ganadería \\
\hline C9 & Depuración natural de aguas \\
\hline C10 & Divulgaciónen los medios masivos de comunicación \\
\hline C11 & Reconocimiento leqal yacadémico \\
\hline C12 & Aqua para uso Industrial \\
\hline C13 & Actividades educativas y científicas \\
\hline C14 & Turismoy recreación \\
\hline C15 & Identidad e intercambio y diversidad cultural \\
\hline C16 & Recursos minerales \\
\hline C17 & Hidroeléctricidad \\
\hline C18 & Defensas vamenazas naturales. \\
\hline C19 & Patrimonio palentológlco \\
\hline C20 & Area de influencia \\
\hline C21 & Vías de comunicación y telecomunicaciones \\
\hline C22 & Límites naturales y referente de regionalización \\
\hline C23 & Diversidad natural \\
\hline C24 & Slngularidad \\
\hline C25 & Interacciones entre ecosistemas \\
\hline
\end{tabular}


Al comparar las valoraciones hechas por los pobladores del parque natural, con las realizadas por los habitantes de la subregión centro del departamento del Huila (Olaya et al., 2003) y con las expuestas por Olaya, Vargas y Rubiano (2005), se observa la tendencia nivel departamental de valorar el parque natural por su servicio hidrológico, junto con las funciones eco sistémicas asociadas a la diversidad natural y el desarrollo cultural de las poblaciones, a diferencia de los pobladores del Parque, quienes lo valoran en función del uso directo y práctico que hacen de los recursos naturales a su disposición.

La comparación expuesta reitera que los pobladores del PNCPM le dan poca importancia en aspectos relacionados con sus funciones eco sistémicas, situación atribuible al desconocimiento que prevalece respecto a la biodiversidad allí presente, de este modo, esta condición se constituye en uno de los principales problemas que se presentan en la gestión del parque natural. Cabe anotar que es una realidad también presente en los habitantes de la cabecera municipal de Garzón.

De igual manera, los diversos funcionarios entrevistados, así como también los funcionarios del sector educativo, reconocen la educación ambiental como factor esencial para lograr la conservación del área protegida y advierten la necesidad de generar mayor conocimiento; sin embargo, no existen investigaciones al respecto, así como tampoco existen programas de capacitación. En consecuencia, el reconocimiento de la importancia eco sistémica, social y económica del parque natural por parte de los habitantes de la Subregión centro del departamento del Huila es bajo.

De manera particular, aunque no existen actividades, o proyectos turísticos, ni se cuenta con la infraestructura adecuada, los pobladores del parque natural plantean la posibilidad de desarrollar actividades turísticas como mecanismo de desarrollo alternativo con tres fines principales: la valoración del parque por ellos mismo y los habitantes de la cabecera municipal, que la actividad turística se convierta en un mecanismo de conservación, y que sea una alternativa para su sustento económico.

En cuanto a la participación comunitaria existe una clara contradicción entre la percepción de los pobladores del parque natural y los funcionarios de las entidades. Mientras que los funcionarios argumentan la vinculación directa y continua de dichos pobladores en todos los proyectos y actividades tendientes a la conservación, éstos disienten, al argumentar que su participación real es inexistente y señalan que sólo se limita a reuniones, lo cual, les impide entender cuál es su papel dentro de lo que se está realizando. $\mathrm{La}$ anterior intencionalidad de los pobladores se constituye en un factor potencial para la conservación tan pronto exista claridad sobre las opciones de manejo y oportunidades que brinda el PNCPM.

También se halló una contradicción relacionada con los efectos de la declaratoria del área protegida en la economía de los habitantes locales. Las instituciones y sus funcionarios coinciden en señalar que el Parque no es una fuente laboral importante, pero los pobladores expresan que la declaratoria ha perjudicado sus ingresos económicos derivados básicamente de la imposibilidad de realizar explotaciones ganaderas y de la extracción de madera. En este orden de ideas, los pobladores creen justo que ellos deben recibir algún tipo de compensación por ayudar a conservarlo.

Pero también hay concordancias en cuanto a la necesidad de realizar conversión tecnológica de los sistemas productivos, en la necesidad de hacer investigación, en la falta de formación ambiental en los pobladores, en el acompañamiento productivo y apoyo en la comercialización de sus productos; en este sentido, la inexistencia de una cadena de valor de productos verdes provenientes del PNCPM dificulta su gestión sostenible, y por último en la necesidad de establecer mecanismos de compensación para los pobladores del parque natural.

\section{Problemas}

En consecuencia se identificaron y definieron 10 problemas $\left(\mathrm{P}_{\mathrm{i}}\right)$, que se relacionan a continuación:

- Oportunidades de desarrollo sostenible no aprovechadas $\left(\mathrm{P}_{1}\right)$.

- Desconocimiento de la importancia ecosistémica, económica, y social de la biodiversidad presente en el PNPM $\left(\mathrm{P}_{2}\right)$.

- Baja articulación entre la CAM, el departamento del Huila, el municipio de Garzón, el sector educativo, las ONG's ambientales y los habitantes de la vereda Las Mercedes $\left(\mathrm{P}_{3}\right)$.

- Deficiente manejo de las fincas compradas para restauración y conservación $\left(\mathrm{P}_{4}\right)$.

- Desprotección de zonas de recarga y nacederos $\left(\mathrm{P}_{5}\right)$. - Pérdida de biodiversidad $\left(\mathbf{P}_{6}\right)$.

- Las necesidades básicas insatisfechas de los habitantes de la vereda Las Mercedes ejercen fuerte presión sobre los bienes y servicios ambientales del $\operatorname{PNPM}\left(\mathrm{P}_{7}\right)$.

- La declaratoria del área protegida ha perjudicado la economía de los habitantes de la vereda Las Mercedes $\left(\mathrm{P}_{8}\right)$.

- Las prácticas agrícolas inadecuadas generan problemas ambientales en el PNPM $\left(\mathrm{P}_{9}\right)$.

- La deficiente cultura ambiental dificulta desarrollar acciones de conservación $\left(\mathrm{P}_{10}\right)$.

Los anteriores problemas muestran una tendencia en la gestión que no involucra las interacciones complejas entre cultura y naturaleza y, en general, no tienen en cuenta las necesidades de los pobladores del Parque, de los habitantes de las cabeceras municipales, ni el accionar institucional como la base de los procesos de conservación. Con estos problemas se realizó un diagrama de influencia - dependencia (figura 2), con el 
cual se logró determinar el siguiente orden de prioridad de los mismos:

$1^{\circ}$ Baja articulación entre la CAM, el departamento del Huila, el municipio de Garzón, el Sector Educativo, las ONG's ambientales y los habitantes de la vereda Las Mercedes $\left(\mathrm{P}_{3}\right)$.

$2^{\circ}$ Desconocimiento de la importancia ecosistémica, económica, y social de la biodiversidad presente en el PNPM $\left(\mathrm{P}_{2}\right)$.

$3^{\circ}$ Oportunidades de desarrollo sostenible no aprovechadas $\left(\mathrm{P}_{1}\right)$.

$4^{\circ} \quad$ La declaratoria del área protegida ha perjudicado la economía de los habitantes de la vereda Las Mercedes $\left(\mathrm{P}_{8}\right)$.

$5^{\circ}$ Las necesidades básicas insatisfechas de los habitantes de la vereda Las Mercedes ejercen fuerte presión sobre los bienes y servicios ambientales del PNPM $\left(\mathrm{P}_{7}\right)$.

$6^{\circ} \quad$ La deficiente cultura ambiental dificulta desarrollar acciones de conservación $\left(\mathrm{P}_{10}\right)$.

$7^{\circ}$ Las prácticas agrícolas inadecuadas generan problemas ambientales en el PNPM $\left(\mathrm{P}_{9}\right)$.

$8^{\circ}$ Deficiente manejo de las fincas compradas para restauración y conservación $\left(\mathbf{P}_{4}\right)$.

$9^{\circ} \quad$ Desprotección de zonas de recarga y nacederos $\left(\mathrm{P}_{5}\right)$.

$10^{\circ}$ Pérdida de biodiversidad $\left(\mathrm{P}_{6}\right)$.

Figura 2. Diagrama de influencia - dependencia de los problemas que se presentan en el Parque Natural Páramo de Miraflores

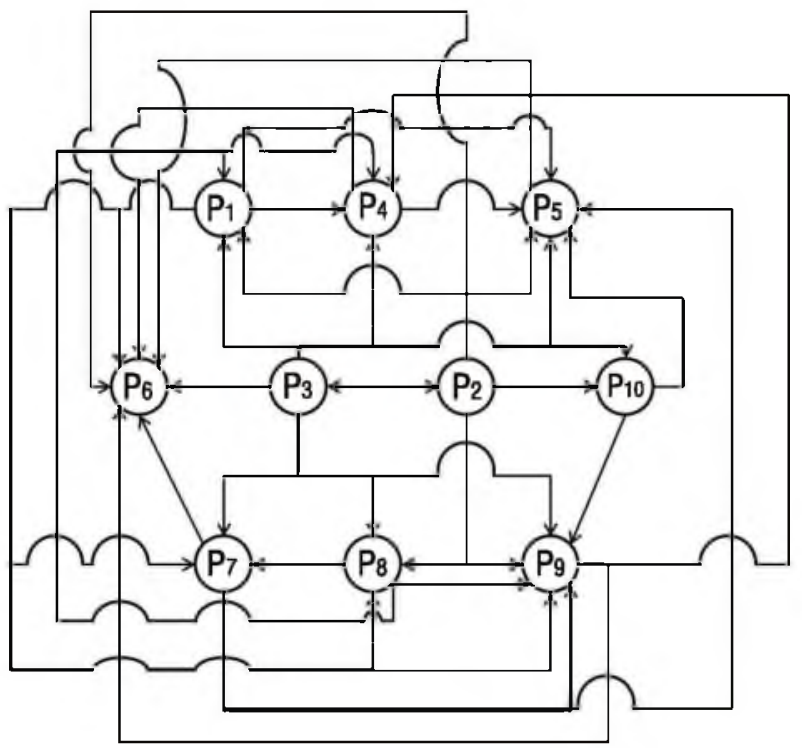

En el diagrama de influencia - dependencia de problemas (figura 2) se evidencia la relación existente entre estos, de modo tal, que se determinan los más relevantes al influir sobre una mayor cantidad de problemas, ya sea de manera directa o indirecta. Los casos contrarios, es decir, los problemas que presentan menor influencia y por ende mayor dependencia son de menor importancia.
De este modo, se puede observar que problemas como la pérdida de biodiversidad $\left(\mathrm{P}_{6}\right)$ y la desprotección de zonas de recarga y nacederos $\left(\mathrm{P}_{5}\right)$, aunque son importantes para la CAM y los municipios de Algeciras, Garzón y Gigante y se constituyen en objetivos de conservación del PNCPM, su solución significativa depende de que primero se solucionen otros problemas, que de manera aparente y por sentido común parecieran ser menos importantes, tales como: La baja articulación entre la CAM, el departamento del Huila, el municipio de Garzón, el sector educativo, las $\mathrm{ONG}^{\prime} \mathrm{s}$ ambientales y los pobladores $\left(\mathrm{P}_{3}\right)$; El desconocimiento de la importancia ecosistémica, económica, y social de la biodiversidad presente en el Parque $\left(\mathbf{P}_{2}\right)$; Las oportunidades de desarrollo sostenible no aprovechadas $\left(\mathbf{P}_{1}\right)$; La declaratoria del área protegida ha perjudicado la economía de los habitantes locales $\left(\mathrm{P}_{8}\right)$; y Las necesidades básicas insatisfechas de los mismos $\left(\mathrm{P}_{7}\right)$ (figura 2).

Resulta oportuno aclarar que la relación encontrada entre los problemas identificados, muestra que si se pretende un desarrollo sostenible basado en las características del ecosistema, es necesario comprender las características sociales, no sólo de los pobladores del PNCPM, sino también de los habitantes de las cabeceras municipales y los sistemas productivos que se benefician de sus bienes y servicios ambientales, al igual que las instituciones que forman parte de la estructura administrativa del territorio y de la autoridad ambiental, situación concordante con lo expuesto por Haberl, Gaube, Díaz-Delgado (2009), Nielsen (2007), Escobar (1999) y Zimmermann (1995).

En este orden de ideas, se puede observar que en cuatro de los primeros cinco problemas se encuentra el factor del recurso económico como determinante. Este hecho, está relacionado con lo expuesto por Márquez (2003), quien indica que en la pobreza radican grandes problemas ambientales y que esta pobreza está asociada a ciertas características geográficas que permiten contar con muchos recursos, pero dificultan la generación de riqueza. De igual manera, también justifica lo planteado por el Convenio sobre la Diversidad Biológica (2004), cuando determina en el principio 4 que los programas de gestión de ecosistemas deben incluir un sistema de incentivos para los que conservan los recursos naturales y señale un pago por parte de quienes se benefician de los bienes y servicios ambientales.

Con referencia a las razones anteriores, los cinco primeros problemas son los más relevantes para la gestión del área protegida, puesto que su solución contribuye a subsanar de manera directa o indirecta los demás problemas, por lo tanto, deben tener especial atención por parte de las autoridades ambientales y territoriales para el mejoramiento del plan de manejo. 


\section{Conclusiones}

La combinación de métodos de Investigación Acción Participación con técnicas de identificación y jerarquización de problemas que se aplicaron en el presente estudio, contribuyó al logro de los objetivos propuestos y fueron coherentes con el enfoque sistémico y validó su potencial uso en el avance hacia la gestión incluyente de las Áreas Protegidas pobladas.

Se determinó que la baja articulación entre las instituciones que forman parte del Sistema Regional de Áreas Protegidas del Huila, la administración municipal, las organizaciones sociales y el sector educativo se constituye en un relevante problema que dificulta lograr la conservación del Parque. En este sentido, se demostró que los problemas que se intuyen como importantes como son la pérdida de biodiversidad y la desprotección de las zonas de recarga acuífera, resultan ser dependientes de problemas de carácter administrativo, sociales, económicos y educativos. En consecuencia, se observa una tendencia en la gestión del parque natural que no involucra las interacciones complejas entre cultura y naturaleza, así como tampoco integra las necesidades de sus pobladores, de los habitantes de las cabeceras municipales, ni el accionar institucional como la base de los procesos de conservación.

En consecuencia, las diferencias contrastantes que existen entre las percepciones ambientales, de los pobladores del PNCPM con la autoridades ambientales y territoriales, las organizaciones sociales y el plan de gestión propuesto para su administración, junto con el bajo interés del sector educativo inciden de manera directa sobre los problemas ambientales que se presentan y disminuyen las posibilidades de conservación sostenibles hacia el futuro.

Teniendo como base de análisis el enfoque por ecosistemas que propone el Convenio sobre la Diversidad Biológica, se logra observar que tanto de parte de los pobladores como de los demás agentes involucrados en la problemática del parque natural existe una necesidad social por controlar la explotación de los recursos naturales allí presentes, pero que hay dificultades para integrar los principios ecológicos con el desarrollo económico puesto que existe un incipiente conocimiento de las realidades sociales, económicas, políticas y ecológicas del PNCPM.

Por tanto, para alcanzar la conservación sostenible del PNCPM y aumentar las posibilidades de éxito en los procesos de gestión ambiental, corresponde a la sociedad y a las autoridades ambientales reorientar las políticas de conservación, hacia la inclusión de los pobladores del parque natural como uno de los pilares para su gestión sostenible.

Los habitantes del parque aprecian el valor de los recursos naturales pero al tiempo este valor ambiental es amenazado por la búsqueda de su sustento económico que lleva a la perdida de la biodiversidad y sus funciones eco sistémicas; es así, que la ejecución de un programa de formación comunitaria, el desarrollo de tecnologías legítimas para el aprovechamiento del área protegida, y la cobertura de las necesidades básicas insatisfechas deben ser una prioridad. En este sentido el turismo cuidadosamente planeado y controlado, junto con el establecimiento de un sistema de pago por servicios ambientales pueden constituirse en alternativas válidas que permitan la conservación del área protegida en el tiempo.

\section{Referencias Bibliográficas}

ALCALDIA MUNICIPAL DE GARZON. (2007). Plan básico de ordenamiento territorial 2007. Garzón: Municipio de Garzón.

Arango, N., Matallana, C., y Puyanna, J. (2005). Planeación regional para la conservación de la biodiversidad. En N. Arango, Bases para el diseño de sistemas regionales de áreas protegidas (págs. 15-28). Bogotá: Instituto de Investigaciones de Recursos Biológicos Alexander von Humboldt.

Bertoldi, S., Fiorito, M., y Álvarez, M. (2006). Grupo Focal y Desarrollo local: aportes para una articulación teórica-metodológica. Ciencia, Docencia y Tecnología(33), 111-131.

CAM, Corporación Autónoma Regional del Alto Magdalena. (2005). Ácuerdo 012 de 2005, Mayo 27, por el cual se declara el Cerro Páramo de Miraflores como Parque Natural Regional y se dictan normas para su administración y manejo sostenible. Neiva: CAM.

CAM, Corporación Autónoma Regional del Alto Magdalena. (2006). Ácuerdo 022 de 2006, Noviembre 30 , por el cual se modifica el acuerdo 012 del 27 de mayo de 2005, que declara el Cerro Páramo de Miraflores como Parque Regional Natural y se dictan normas para su administración y manejo sostenible. Neiva: CAM.

Cardona, N. (2005). Consideraciones socioeconómicas en el diseño de proyectos sustentables de restauración ecológica. En $O$. Sánchez, E. Peters, R. Marquéz, E. Vega, G. Portales, M. Valdez, y otros, Temas sobre restauración ecológica (págs. 45-66). Mexico D.F.: Instituto Nacional de Ecología.

Castillo, A. (2005). Comunicación para la restauración: perspectiva de los actores en intervenciones con y por medio de las personas. En O. Sánchez, E. Peters, R. Marquéz, E. Vega, G. Portales, M. Valdez, y otros, Temas sobre restauración ecológica (págs. 67-78). Mexico: Instituto Nacional de Ecología.

CDB, Convenio Sobre la Diversidad Biológica. (2004). Enfoque por Ecosistemas. Recuperado el 20 de Diciembre de 2010, de Convenio sobre la Diversidad Biológica: http://www.cbd.int/doc/publications/ea-text-es.pdf

COLOMBIA. CONSEJO MUNICIPAL DE GIGANTE. (2000). Acuerdo 035 de 2000, diciembre 6, por el cual se adopta el Esquema de Ordenamiento Territorial del Municipio de Gigante. Gigante: Consejo Municipal de Gigante.

COLOMBIA. MINISTERIO DE AMBIENTE VIVIENDA Y DESARROLLO TERRITORIAL. (2010). Decreto 2372: En relación con el Sistema Nacional de Areas Protegidas, las categorías de manejo que lo conforman y se dictan otras disposiciones. Bogotá, D.C.: MVDT.

Escobar, A. (1999). El Final del Salvaje: Naturaleza, Cultura y Política en la Antropología Contemporanea. Bogotá: CEREC - ICAN.

Fernández, Y. (2008). ¿Por qué estudiar las percepciones ambientales? Una revisión de la literatura mexicana con énfasis en Áreas Naturales Protegidas. Espiral, XV.

Gallego, B. R. (2000). El problema de las competencias cognoscitivas: Una discusión necesaria. Santafé de Bogotá D.C.: Universidad Pedagógica Nacional.

González, F., y Galindo, M. (1999). Ambiente y Desarrollo: Ensayos II, Elementos para la cosideración de la dimensión ético-política en la valoración y uso de la biodiversidad. Bogotá: Pontificia Universidad Javeriana, Instituto de Estudios Ambientales para el Desarrollo IDEADE.

Haberl, H., Gaube, V., Díaz-Delgado, R., Krauze, K., Neuner, A., Peterseil, J., y otros. (2009). Towards an integrated model of socioeconomic biodiversity drivers, pressures and impacts. A feasibility study based on three European long-term socioecological research platforms. Ecological Economics, 68(6), 17971812 .

Ingenieros y Biólogos Ltda. (2006). Actualización del plan de manejo Parque Natural Regional Cerro Páramo de Miraflores. Neiva. 
Instituto para la Sostenibilidad del Desarrollo. (2007). Análisis y Diseño de Mecanismos Financieros de Âreas Protegidas Regionales y Locales: Bases para la Estrategia de Sostenibilidad Financiera del Sistema Departamental de Areas Protegidas del Huila. Bogotá: Patrimonio Natural. Fondo para la Biodiversidad y Áreas Protegidas.

Kallbekken, S., y Aasen, M. (2010). The demand for earmarking: Results from a focus group study. Ecological Economics, 2183-2190.

Kattan, G. (2005). Planificación del Eden: Principios Fundamentales en el Diseño de sistemas Regionales de Áreas Protegidas. En N. Arango, Bases para el Diseño de Sistemas Regionales de Áreas Protegidas (págs. 51-80). Bogotá: Instituto de Investigaciones de Recursos Biológicos Alexander von Humboldt.

Márquez, G. (2003). Ecosistemas Estratégicos de Colombia. Recuperado el 17 de Febreo de 2011, de Sociedad Geográfica de Colombia: http://www.sogeocol.edu.co/documentos/07ecos.pdf

Martínez, Z. (2004). Cogestión de recursos naturales en la Región Atlántica de Colombia. Innovar (On line), 14(23), 158-167.

Morales, R., Otero, M., García, J., Hammen, T. v., y Torres, A. (2007). Atlas de páramos de Colombia. Bogotá D.C.: Instituto de Investigación de Recursos Biológicos Alexander Von Humboldt.

Nielsen, S. (2007). What has modern ecosystem theory to offer to cleaner production, industrial ecology and society? The views of an ecologist. Journal of Cleaner Production, 15(17), 1639-1653.

Olaya, A. (2003). Sistema de apoyo para la toma de decisiones en distritos de riego y drenaje a partir de sus recursos, restricciones impactos ambientales para el caso Colombia, Tesis (Doctor en Ingeniería Área Recursos Hidráulicos). Medellín: Universidad Nacional de Colombia sede Medellín. Facultad de Minas. Escuela de Geociencias y Medio Ambiente. Postgrado en Aprovechamiento de Recursos Hídricos.

Olaya, A., y Sánchez, M. (2005). Significado ecológico y sociocultural de los ecosistemas estratégicos del Huila. En A. Olaya, y M. Sánchez, Del Macizo Colombiano al Desierto La Tatacoa: La ruta del río Magdalena en el Huila (págs. 17-29). Neiva: Universidad Surcolombiana.

Olaya, A., Sánchez, M., y Sogamoso, E. (2003). Ecosistemas estratégicos del Huila por subregiones, según la percepción de diferentes actores sociales. En A. Olaya, y M. Sánchez, Ecosistemas Estratégicos del Huila: Significado ecológico y cultural (págs. 322344). Neiva: Universidad Surcolombiana.
Olaya, A., Vargas, C., y Rubiano, G. (2005). Cerro Páramo de Miraflores, primer Parque Natural Regional declarado en el departamento del Huila. En A. Olaya, y M. Sánchez, Del Macizo Colombiano al Desierto La Tatacoa: La ruta del río Magdalena en el Huila (págs. 271-286). Neiva: Universidad Surcolombiana.

Powe, A., Garrod, G., y McMahon, P. (2005). Mixing methods within stated preference environmental valuation:- Ecological Economics, 52, 513-526.

RECUPERAM Ltda. (2003). Caracterización ambiental, social y económica del ecosistema estratégico del Cerro de Miraflores y formulación del plan de manejo ambiental para su declaratoria como área natural protegida. Neiva: CAM.

Ressel, L., Colomé, C., Gualda, D., Hoffmann, L, Marion da Silva, D., Y Dutra, D. (2008). O USO DO GRUPO FOCAL EM PESOUISA OUALITATIVA. Texto Contexto Enferm, Florianópolis, 17(4), 779-786.

Salazar, N., Lara, G., Guido, S., Toro, I., y Obando, L. (2000). Comunicación aumentativa y alternativa. Santa Fe de Bogotá, D.C.: Universidad Pedagógica Nacional.

Scott, A. (2011). Focussing in on focus groups: Effective participative tools or cheap fixes for land. Land Use Policy, 1-11.

Sheil, D., Puri, R., Basuki, I., Heist, M., Wam, M., Liswanti, N., y otros. (2004). Center for International Forestry Research CIFOR. Recuperado el 14 de junio de 2010, de Explorando la biodiversidad, el medio ambiente y las perspectivas de los pobladores en áreas boscosas: Métodos para la valoración multidisciplinaria del paisaje: http://www.cifor.cgiar.org/nc/online-library/browse/ view- publication/publication/1616.html

Vásquez, F. (2007). Reconceptualizando la biodiversidad en América Latina: Una propuesta biocultural y territorial con visión de futuro. Revista Virtual REDESMA "Red de Desarrollo Sostenible y Medio Ambiente", 1, 38-47.

White, R., Fischer, A., Marshall, K., Travis, J., Webb, T., Falco, S., y otros. (2009). Developing an integrated conceptual framework to understand biodiversity conflicts. Land Use Policy, 26(2), 242-253.

Xenariosa, S., y Tziritis, I. (2007). Improving pluralism in Multi Criteria Decision Aid approach. Ecological Economics, 62, 692-703.

Zambrano, J. (2007). Sistemas productivos en el Macizo Colombiano. En CAM, Memorias II expedición al Macizo Colombiano (págs. 4354). Neiva: CAM

Zimmermann, M. (1995). Psicología ambiental y calidad de vida. Bogota: Universidad Nacional de Colombia. 